\title{
Traqueobroncomalacia en niños
}

\author{
Dr. José Perillán'
}

I Hospital San Juan de Dios. Universidad de Chile. Universidad Finis Terrae.

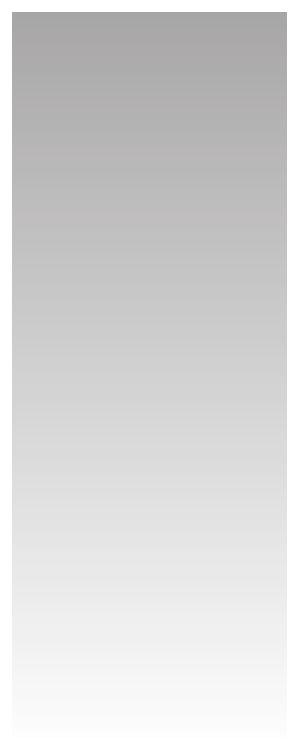

\section{TRACHEOBRONCHOMALACIA IN CHILDREN}

Tracheobronchomalacia refers to the presence of trachea and bronchi with soft, collapsible walls. Its incidence has been reported from I: I 500 to 1:2500. It can be congenital or acquired. This disease ranges from mild to life threatening. A high clinical suspicion is required for diagnosis, which needs to be confirmed endoscopically. Depending on severity, treatment can include from physical therapy and antibiotics to mechanical ventilation, surgery and tracheostomy, and is based on expert opinion and case series. Prognosis is usually good, with tendency to spontaneous resolution near the $2 d$ year of life.

Key words: Tracheobronchomalacia, bonchoscopy, tracheostomy, aortopexy, children.

\section{RESUMEN}

Traquebroncomalacia se refiere a la presencia de tráquea y/o bronquios cuya pared es blanda y tiene tendencia al colapso. Se ha reportado una incidencia entre 1 : I.500 a 1:2.500. Puede ser congénita o adquirida. La severidad de los síntomas varía desde leve a incluso riesgo vital y muerte. El diagnóstico requiere una alta sospecha clínica y confirmación endoscópica. El tratamiento está basado en opiniones de expertos y series clínicas y varía según la severidad de los síntomas desde kinesioterapia y antibióticos en los casos más leves hasta ventilación mecánica, traqueostomía y cirugía en los más severos. El pronóstico en general es bueno con tendencia a la resolución espontánea aproximadamente a los 2 años de vida.

Palabras clave: Traqueobroncomalacia, broncoscopía, traqueostomía, aortopexia, niños.

\section{DEFINICIÓN}

Malacia deriva del griego "malakia" que significa "blando". Aplicado a medicina se refiere habitualmente a huesos o cartílagos. Traqueomalacia (TM) se define como una debilidad de la pared traqueal, secundaria a una reducción y/o atrofia de las fibras elásticas longitudinales de la pars membranosa o a un daño en la integridad del cartílago. A consecuencia de esto la pared es blanda por lo que la vía aérea tiene tendencia al colapso (Figura I). Normalmente la vía aérea intratorácica tiende a colapsar en espiración y a dilatarse en inspiración, mientras que la extratorácica tiende a colapsar en inspiración y dilatarse en espiración. Al haber malacia estos fenómenos fisiológicos se agravan(l).

La TM se localiza más frecuentemente a nivel intratorácico por lo que la principal manifestación es la obstrucción al flujo aéreo en espiración. La TM puede comprometer una porción de la tráquea o incluso ser total. Si además hay compromiso bronquial hablamos de traqueobroncomalacia (TBM). Broncomalacia (BM) es la debilidad y tendencia al colapso de uno o más bronquios principales sin compromiso traqueal. Es mucho menos frecuente que la TM y la TBM.

Correspondencia: Dr. José Perillán T. Hospital San Juan de Dios.

Universidad de Chile. Universidad Finis Terrae.

E-mail: joseperillan@med.uchile.cl
El diagnóstico de las malacias de la vía aérea ha aumentado significativamente los últimos años en relación a una mayor sospecha clínica y a un mayor acceso a técnicas de imágenes y endoscópicas; sin embargo, aún hay poca claridad respecto al tratamiento más adecuado.

\section{CLASIFICACIÓN}

\section{Primaria o congénita}

La TM es la forma más común de anomalía traqueal congénita. Es más frecuente en prematuros pero también se puede encontrar en niños sanos. Se piensa que es secundaria a una inmadurez del cartílago traqueal. Algunos autores han reportado mayor riesgo en el sexo masculino mientras otros no reportan diferencias de género. Se incluyen en este grupo aquellos procesos patológicos que resultan en la formación de una matriz cartilaginosa anormal como policondritis y condromalacia, que terminan en una alteración en la maduración de las fibras de colágeno y una debilidad de los tejidos traqueobronquiales. Además la TM está claramente asociada con la mucopolisacaridosis, como el síndrome de Hunter y el síndrome de Hurler, así como con otros síndromes genéticos (Tabla I).

La asociación más común es la fístula traqueoesofágica (FTE). En 75\% de autopsias de niños con FTE o atresia esofágica (AE) se encuentra traqueomalacia. Algunos autores consideran que la TM sería un estadio inicial del mismo 


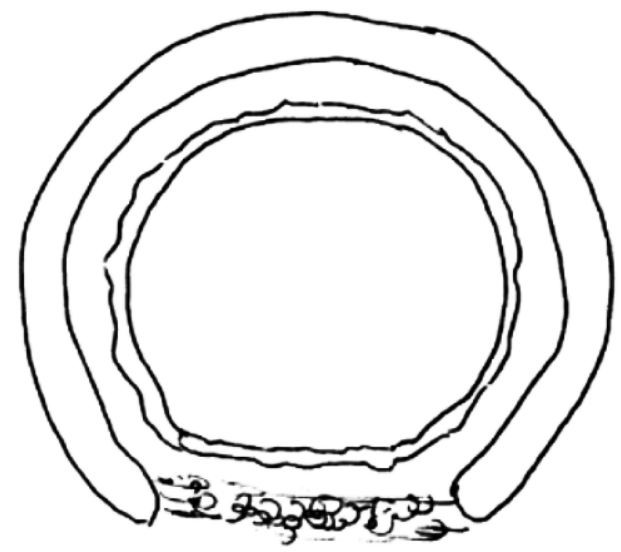

Tráquea normal

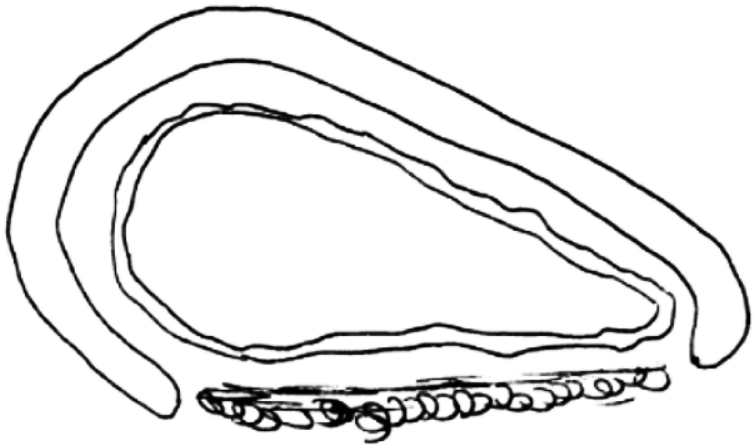

Traqueomalacia

Figura I.

Tabla I. Condiciones asociadas a traqueobroncomalacia

\section{Primarias (congénitas)}

Recién nacidos sanos (TM primaria o idiopática)

Prematurez

Colapso pulsátil con arteria innominada normal

Anormalidades congénitas del cartílago

Discondroplasia/condromalacia/condrodisplasia

Policondritis

Síndrome de Ehlers-Danlos

Síndromes congénitos asociados con TM y TBM

Mucopolisacaridosis

Síndrome de Hurler

Síndrome de Hunter

Asociación CHARGE

Asociación VACTERL

Trisomía 9

Trisomía 21

Atelosteogenesis tipo I

Síndrome de Antley-Bixler

Deleción IIp|3

Deleción 22q I I

Translocación 18-22

Síndrome de Hallermann-Streiff

Síndrome de Pfeiffer

Síndrome de Blackfan-Diamond

Síndrome de Williams-Campbell

Displasia de Kniest

Síndrome de Di George

Deleción de 12q

Síndrome de Larsen y síndromes tipo Larsen

Síndrome de Brachmann-De Lange

Displasia Camptomélica

Síndrome de Pierre-Robin

Anomalías congénitas asociadas con TM y TBM

Fístula traqueoesofágica

Atresia esofágica con o sin hendidura laríngea

Displasia broncopulmonar

\section{Secundarias (adquiridas)}

Intubación prolongada

Traqueostomía

Traqueobronquitis severa

Condiciones asociadas a compresión

Vascular

\section{Doble arco aórtico}

Inserción anormal de la arteria innominada

Anomalía de la arteria pulmonar izquierda

Arco aórtico derecho

Subclavia derecha aberrante

Venas pulmonares dilatadas

Cardíacas

Hipertrofia de aurícula izquierda

Dilatación de aurícula izquierda

Esqueléticas

Escoliosis

Pectus excavatum

Tumores y quistes

Teratoma

Higroma quístico

Hemangioma

Quiste broncogénico

Quiste enterogénico

Timoma

Timo hipertrófico

Bocio

Malformación linfática

Linfoma

Neuroblastoma

Infección

Abceso

Post-traumática 
proceso patológico embrionario que lleva a la producción de una FTE o una AE. En la mayoría de los casos la TM primaria es autolimitada, produciéndose normalización de la estructura traqueal a los 2 años de edad. En los pacientes con síndromes malformativos y enfermedades del colágeno el problema tiende a persistir y ser más grave, incluso fatal.

\section{Secundaria o adquirida}

Se producen por degeneración de un cartílago traqueal normal por diversas causas. Es más frecuente que la TM primaria. Es más frecuente en hombres, si tener esto una explicación clara. Es común que se produzca por una intubación endotraqueal prolongada, por injurias múltiples como toxicidad por oxígeno, infecciones recurrentes y aumento de presión de la vía aérea. El riesgo es mayor en prematuros con síndrome de dificultad respiratoria por la inmadurez de los tejidos. La traqueostomía también favorece el desarrollo de traqueomalacia, no sólo en la zona de ostomia sino también en relación al cuff y a la punta de la cánula, probablemente por necrosis por presión, compromiso de la perfusión, infecciones y daño mucoso por fricción. Además la traqueostomía se asocia con traqueobronquitis recurrente.

Una proporción menor de TM es causada por compresiones externas a la tráquea (Tabla I). La compresión afecta la integridad de la pared traqueal y aumenta la compliance de los tejidos traqueales adyacentes a la obstrucción. Aunque algunas lesiones son lo suficientemente severas como para causar obstrucción persistente de la vía aérea, muchas causan sólo un colapso transitorio en relación a aumentos de presión intratorácica. Las estructuras implicadas en el desarrollo de TM secundaria por compresión incluyen cardiopatías como doble arco aórtico, rama anormal de la arteria innominada, otros anillos vasculares, anomalías de la arteria pulmonar izquierda, arco aórtico derecho con ligamento arterioso izquierdo e hipertrofia de aurícula izquierda. Alteraciones esqueléticas, como escoliosis o pectus excavatum, pueden causar TM secundaria. Lesiones que efecto de masa, como bocio, tumores, quistes y abscesos, también pueden comprimir la tráquea. Aún después de la corrección quirúrgica de la lesión la debilidad de la pared traqueal puede persistir y requerir tratamiento adicional.

\section{Condiciones asociadas}

Hay muchas condiciones asociadas a TM. Cardiopatías se encuentran en el 20-58\% de pacientes con TM. Estas incluyen ductus arterioso persistente, defectos de septum interauricular o interventricular, alteraciones del arco aórtico, hipoplasia de corazón izquierdo, hipoplasia de corazón derecho, tetralogía de Fallot, dextrocardia y estenosis valvulares. Hasta el 52\% de los pacientes con TM tienen asociada displasia broncopulmonar. Aproximadamente la mitad tienen reflujo gastroesofágico y hasta $78 \%$ de aquellos con TM de riesgo vital, requiriendo fundoplicatura junto al manejo de la TM.

Se puede asociar también a estenosis subglótica, laringomalacia y parálisis de cuerdas vocales. La TM también se puede producir en el contexto de un sistema nervioso autónomo inmaduro. Daño neurológico se ha descrito en 8-48\% de lactantes con TM y en $26 \%$ de los casos más severos.

\section{PATOGENIA}

La patogenia no es bien conocida. A través de evidencia indirecta se estima que el trastorno se produce aproximadamente entre las 5 a 8 semanas de gestación. Se han identificado varios genes que podrían estar involucrados, incluyendo los genes Hox, Shh y Gli. El gen Sox9 es esencial en la formación del cartílago tanto espacial como temporalmente y la expresión del gen Shh es importante para la formación de los anillos cartilaginosos.

Alteraciones en el momento de expresión o en los componentes que dan soporte estructural al cartílago pueden alterar las propiedades mecánicas del cartílago traqueal. El colágeno tipo II tiene el rol dominante en la tensión del cartílago. El colágeno tipo I tiene un rol más importante en la formación de la vía aérea. La distribución anatómica de los diversos tipos de colágeno en la vía aérea complica le asunto, porque los patrones de distribución de los colágenos tipo I, II y III cambian temporal y regionalmente desde el período neonatal a la infancia. Aun no se sabe cómo la expresión génica controla esta transición, pero alteraciones en estos procesos podrían llevar a una vía aérea vulnerable temporal y espacialmente y al desarrollo de malacia ${ }^{(2)}$.

\section{HISTOPATOLOGÍA}

Se describe en autopsias un aumento del perímetro traqueal interno y del largo del cartílago comparado con controles, la membrana traqueal es más grande y la proporción de cartílago y músculo están disminuidos lo que explicaría la tendencia al colapso. Se ha encontrado que la musculatura transversa está consistentemente conservada, en cambio hay gran variabilidad en las fibras longitudinales. Estas fibras tendrían un rol importante en evitar el colapso especialmente en la tráquea intratorácica. Los prematuros tienen la menor proporción de estas fibras (31\%) siendo normal en los mayores de un año (54\%). La anormalidad más común es una deficiencia en el cartílago junto a un mayor largo de la membrana muscular. Hay una disminución en la proporción normal de cartílago (4.5: I) en 68\% de pacientes con FTE.

Mair y Parsons proponen una clasificación según histología y endoscopia. Tipo I sería un colapso traqueal congénito sin obstrucción de la vía aérea. Correspondería a prematuros, lactantes con AE, FTE, mucopolisacaridosis y síndrome de Larsen. Tipo II serían aquellos con colapso traqueal secundario a una compresión extrínseca como cardiopatías, quistes, tumores o bocio. Tipo III serían aquellas TM secundarias a intubación prolongada, traqueostomía o traqueobronquitis severa.

\section{INCIDENCIA E HISTORIA NATURAL}

La incidencia de TBM se ha reportado entre 1:1.500 a 1:2.500 niños; sin embargo, se estima que hay un importante subdiagnóstico. Hay reportes de TM en 15\% de 664 broncoscopias. En otro estudio en menores de 3 años con dificultad respiratoria se encontró TM en $30 \%$. En un estudio 
Tabla 2. Síntomas de traqueomalacia

\begin{tabular}{|l|}
\hline Estridor \\
\hline Tos perruna \\
\hline Dificultad respiratoria \\
\hline Sibilancias \\
\hline Apnea refleja \\
\hline Cianosis \\
Bradicardia \\
\hline Taquiarritmia \\
\hline Hiperextensión espontánea del cuello \\
\hline Prolongación de la fase espiratoria \\
\hline Falla de crecimiento \\
Aumento de trabajo respiratorio \\
Retracción esternal, subesternal e intercostal \\
Infecciones respiratorias recurrentes \\
Paro respiratorio \\
Paro cardíaco \\
\hline
\end{tabular}

de 50 lactantes con TM el $48 \%$ fue considerado primaria y el 52\% secundaria; $96 \%$ de lactantes con TM secundaria eran prematuros con ventilación mecánica prolongada. Con el desarrollo de los cuidados intensivos neonatales y la broncoscopia ha aumentado el reporte de TM, sin embargo, el diagnóstico no está bien estandarizado.

La TM se asocia con morbilidad y mortalidad. Puede ser subdiagnosticada o mal diagnosticada como asma u otras enfermedades respiratorias. La severidad puede variar de leve a riesgo vital y la letalidad en los casos graves puede llegar hasta $80 \%$.

\section{SÍNTOMAS}

Clásicamente se ha descrito que los síntomas de TM no se presentan al nacimiento sino que van apareciendo en forma insidiosa durante las primeras semanas o meses de vida. Un estudio más reciente describe que 95\% de TM congénitas presentan síntomas al nacimiento.

Estridor espiratorio y tos "de perro" son los síntomas más reportados (Tabla 2). Si la malacia es de tráquea extratorácica el estridor será inspiratorio. La tos se produce por la yuxtaposición de la pared anterior y posterior de la tráquea, resultando en vibración recurrente e irritación de la misma. Puede haber respiración ruidosa de tonalidad media o alta. Se han reportado también dificultad respiratoria recurrente, sibilancias, cianosis e hiperextensión espontánea del cuello.

Puede haber retracción esternal, subesternal e intercostal. Los síntomas pueden aumentar en relación a esfuerzos como comer, llorar o toser. Suele asociarse un compromiso del clearence mucociliar por lo que hay obstrucción del lumen bronquial durante la tos. Se asocia a infecciones respiratorias y neumonía recurrente. Puede haber dificultad para la alimentación, en especial en aquellos con compresiones vasculares, manifestada como disfagia, tos, cianosis, regurgitación. Esto secundario a fenómenos anatómicos y reflejos. Al llenarse el esófago y ocluir la tráquea se produce obstrucción traqueal e hipoxemia, lo que impide continuar la alimentación, produciendo mal incremento ponderal. Puede ser necesario alimentar al lactante en forma muy fraccionada. Puede ocurrir una "apnea refleja" por obstrucción traqueal por alimentos en el esófago o secreciones en tráquea con letalidad elevada.

Se ha propuesto un puntaje de severidad de síntomas. Los pacientes leves tendrían sólo dificultad respiratoria en relación a infecciones agregadas. Los pacientes moderados presentan estridor, sibilancias, infecciones respiratorias recurrentes e incluso cianosis en relación a exacerbaciones. Los severos presentan estridor de reposo, marcada retención de secreciones, obstrucción de la vía aérea, apnea refleja e incluso paro cardíaco.

\section{DIAGNÓSTICO}

TM y TBM deben ser diferenciados de asma, obstrucción intraluminal, cuerpo extraño y otras enfermedades. Se necesita un alto índice de sospecha. Se ha descrito un retraso en el diagnóstico de 6-8 meses en promedio, con un rango desde I semana hasta varios años. Resulta fundamental una buena anamnesis y examen físico.

Se debe sospechar el diagnóstico en niños con síntomas respiratorios recurrentes o prolongados: tos húmeda persistente o recurrente, tos inusual, estridor espiratorio, sibilancias, disnea o dificultad respiratoria, bronquitis bacteriana prolongada recurrente, neumonía (especialmente con atelectasia recurrente o persistente), atrapamiento aéreo localizado o hallazgos radiológicos inusuales. La sospecha debe ser mayor si se asocia a cardiopatías, bronquiectasias no fibrosis quística y cuadros de "pseudoasma" que no responden a terapia habitual $(\text { grado de evidencia }=>\text { moderado })^{(3)}$.

Las pruebas de función pulmonar pueden ayudar mostrando una curva flujo/volumen con limitación al flujo espiratorio y $\mathrm{FIF}_{50} / \mathrm{FEF}_{50}$ disminuido, sin embargo, requieren la cooperación del paciente por lo que es difícil de aplicar en niños pequeños. La radiografía convencional tiene una sensibilidad del $60 \%$. Los estudios contrastados con bario ayudan en la evaluación de FTE, AE, reflujo gastroesofágico y anillos vasculares (Figura 2) $)^{(4)}$.

La tomografía computada, en especial la helicoidal y multicorte, tiene una alta sensibilidad, permite hacer reconstrucciones $2 \mathrm{D}$ y $3 \mathrm{D}$ y realizar broncoscopia virtual, no es invasiva, pero aún no se ha demostrado que reemplace a la broncoscopia y tiene el problema de la radiación, la que de todas maneras se puede disminuir con protocolos adecuados. Su utilidad es mayor en TM y menor en broncomalacia. Su principal utilidad está en la evaluación de compresiones extrínsecas. En la evaluación de estructuras vasculares puede ser superior la resonancia magnética nuclear, que además tiene la ventaja de no emanar radiación ionizante.

A pesar del avance en las técnicas de imágenes, estas fallan en entregar una visión dinámica de la vía aérea, en espe- 


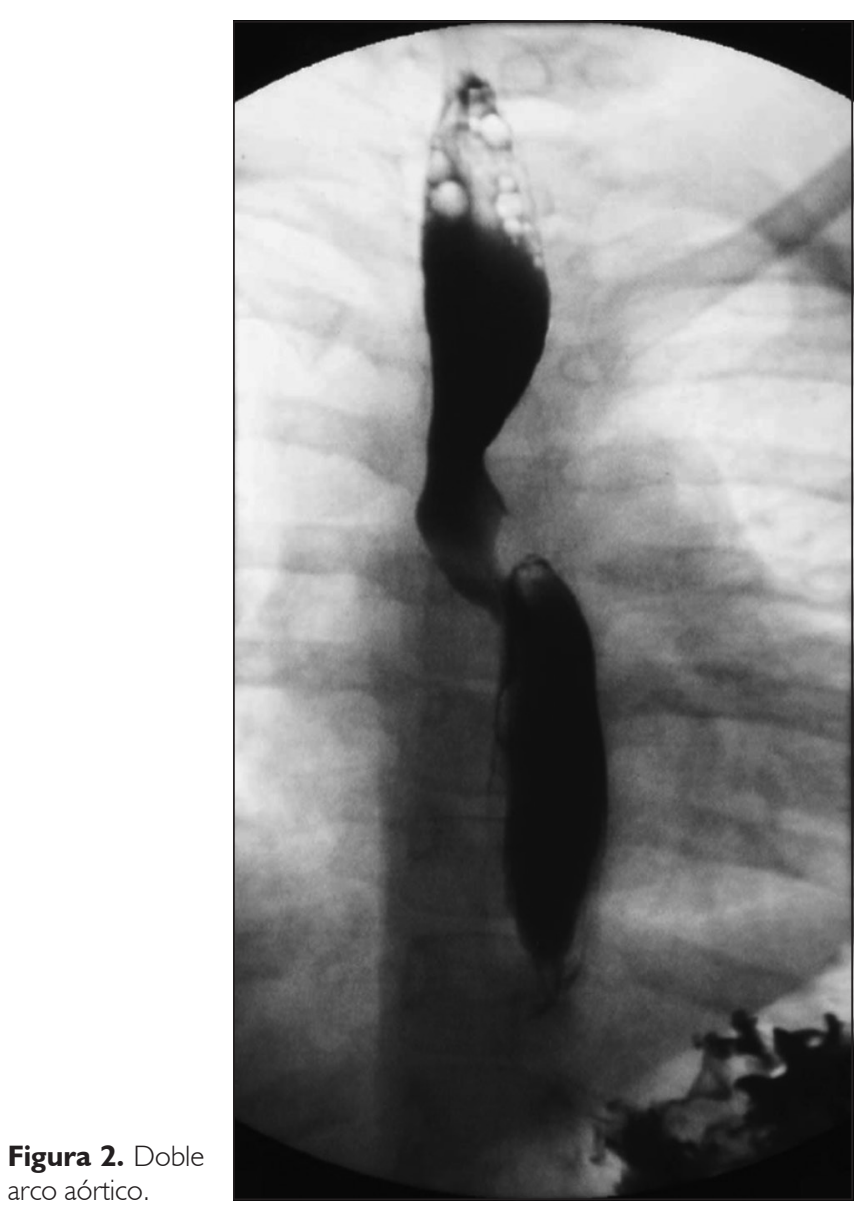

cial en los niños pequeños que no pueden cooperar con el examen, por lo que muchos expertos siguen considerando la endoscopia como fundamental en el diagnóstico. Una dificultad en niños es la necesidad, en algunos casos, de sedación intensa y/o ventilación durante el examen lo que puede hacer que el colapso no se vea. Lo ideal es una anestesia o sedación con ventilación espontánea y sin intubación. Un colapso traqueal de más del 50\% es considerado anormal. La mayoría de los lactantes con traqueomalacia tienen un colapso de más del 75\% y en 33\% puede llegar a ser total (Figura 3).

\section{TRATAMIENTO}

El tratamiento está básicamente basado en opiniones de expertos derivadas de experiencias limitadas usando niveles de severidad basados en síntomas y aspecto endoscópico. Se han desarrollado algoritmos de tratamiento pero ninguno ha sido testeado rigurosamente (nivel de evidencia $=>$ muy bajo). Además el grado de obstrucción de la vía aérea a la endoscopia parece no tener una relación directa con la severidad de los síntomas (nivel de evidencia $=>$ moderado).

Muchos pacientes no necesitan tratamiento, en la medida que el niño crece el cartílago se vuelve más fuerte y duro. En las TM leves y moderadas los síntomas habitualmente se resuelven entre el año y los dos años de vida. Por lo tanto, en los casos leves se recomienda un tratamiento conservador que incluye tratar las infecciones respiratorias, oxígenoterapia y kinesioterapia respiratoria.

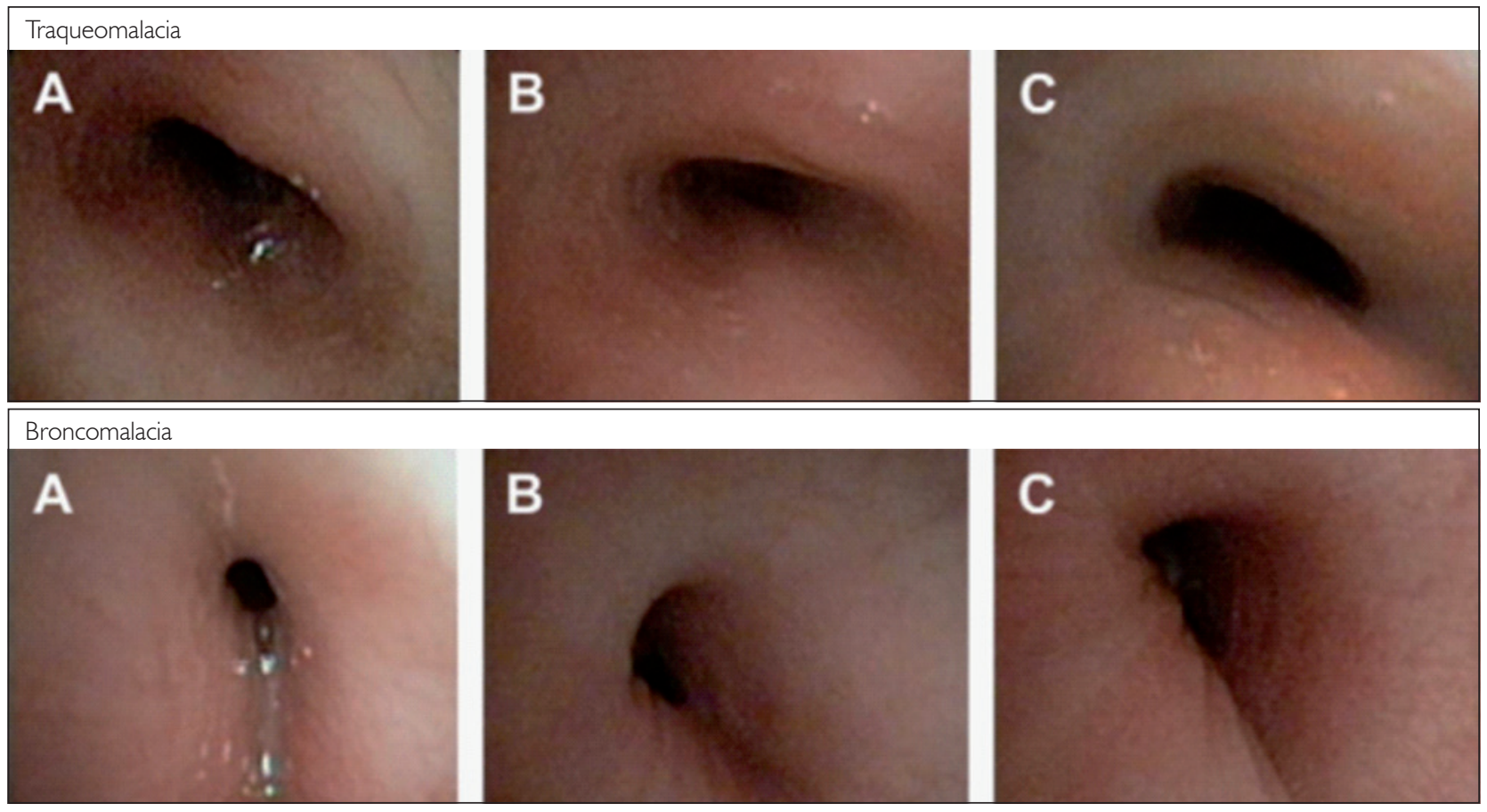

Figura 3. 
La DNAsa usada por 2 semanas en pacientes con TBM e infección respiratoria no ha mostrado acelerar la recuperación o disminuir la necesidad de antibióticos ${ }^{(5)}$.

Para aquellos pacientes que no mejoran o que tienen síntomas de riesgo vital hay varias opciones terapéuticas. La terapia clásica era la traqueostomía (TQT) y ventilación mecánica prolongada. La cánula debía tener el largo suficiente para superar la zona de malacia o agregarse un tubo interno. Los problemas de esta estrategia incluyen la necesidad de cambiar la cánula en la medida que el niño crece, broncoespasmo recurrente, pérdida del mecanismo glótico de aumento de presión de vía aérea y dificultad en la decanulación. Además se agrega injuria traqueal por la cánula e infecciones recurrentes lo que hace que se prefiera otras modalidades terapéuticas de ser posible. El porcentaje de niño que requiere TQT varía entre 12 y 62\%. Otros datos muestran una necesidad de TQT en 75\% de prematuros versus 39\% de niños de término. El 7I\% se pude decanular a los 30 meses sin necesidad de cirugía correctora. El 32\% de niños TQT en domicilio eran por TM en algunas series.

El CPAP es una modalidad efectiva de tratamiento para la TM, TBM y BM moderada a severa. Evita el colapso de la vía aérea durante la respiración funcionando como un "stent neumático". Los inconvenientes del CPAP son la dificultad en la alimentación oral, retraso en el lenguaje y eventualmente retraso del desarrollo psicomotor. El CPAP se ha necesitado por 22 meses en prematuros y 21 meses en lactantes de término. Según la estimación de la duración del tratamiento el CPAP se puede considerar el tratamiento de primera línea para las malacias de la vía aérea o como coadyuvante de otras terapias. Sería la única opción en BM severa.

En pacientes con formas más severas de TM que no responden a tratamiento médico o que presentan síntomas de riesgo vital puede ser necesario un tratamiento quirúrgico. Son indicaciones de cirugía neumonía recurrente, obstrucción respiratoria intermitente y la imposibilidad de extubar. La presencia de apneas reflejas es indicación de hospitalización hasta resolución quirúrgica.

Tradicionalmente la aortopexia ha sido el procedimiento de elección en el tratamiento de la TM severa. Se ingresa a través de una toracotomía por el $3^{\circ}$ espacio intercostal derecho (u otra ruta alternativa) y se fija la pared de la aorta a la pared posterior del esternón lo que tracciona la pared anterior de la tráquea hacia delante. Esto aumenta el diámetro anteroposterior de la tráquea y disminuye el colapso de la misma (Figura 4). La aortopexia no siempre es exitosa (hasta un 95\%) y puede haber complicaciones (lesión vascular, lesión del nervio frénico) por lo que se han evaluado otras opciones quirúrgicas.

Implantes con material autólogo (costilla) o prótesis artificiales se han utilizado para dar soporte a la pared traqueal fláccida. El soporte se puede dar suturando un material rígido a la membrana traqueal a través de una toracotomía posterolateral derecha o envolviendo $3 / 4$ del perímetro traqueal a través de un acceso cervical anterior o una esternotomía media. Es un procedimiento invasivo que puede no resolver bien lesiones distales y no ser bien tolerado en pacientes más complicados. Aparentemente no compromete el crecimiento traqueal. Otras técnicas como traqueopexia, resección traqueal y reconstrucción traqueal han mostrado escaso éxito.

Los stent traqueales internos tienen la ventaja de ser menos invasivos. Pueden ser de silicona o metálicos. Los metálicos tienen la ventaja de ser más delgados y de fácil utilización. Hay series de casos con manejo exitoso en niños, evitando la obstrucción al flujo aéreo y permitiendo la extubación. Sin embargo, la instalación no está exenta de riesgos (incluso muerte) y la aparición posterior de tejido de granulación es una complicación consistente. Otras complicaciones son la dificultad para removerlos, muerte, fractura del stent, necesidad de un stent adicional, migración y necesidad de dilatar el stent cuando el niño crece. La ventaja de los stent de silicona es que es más fácil removerlos e instalar uno más

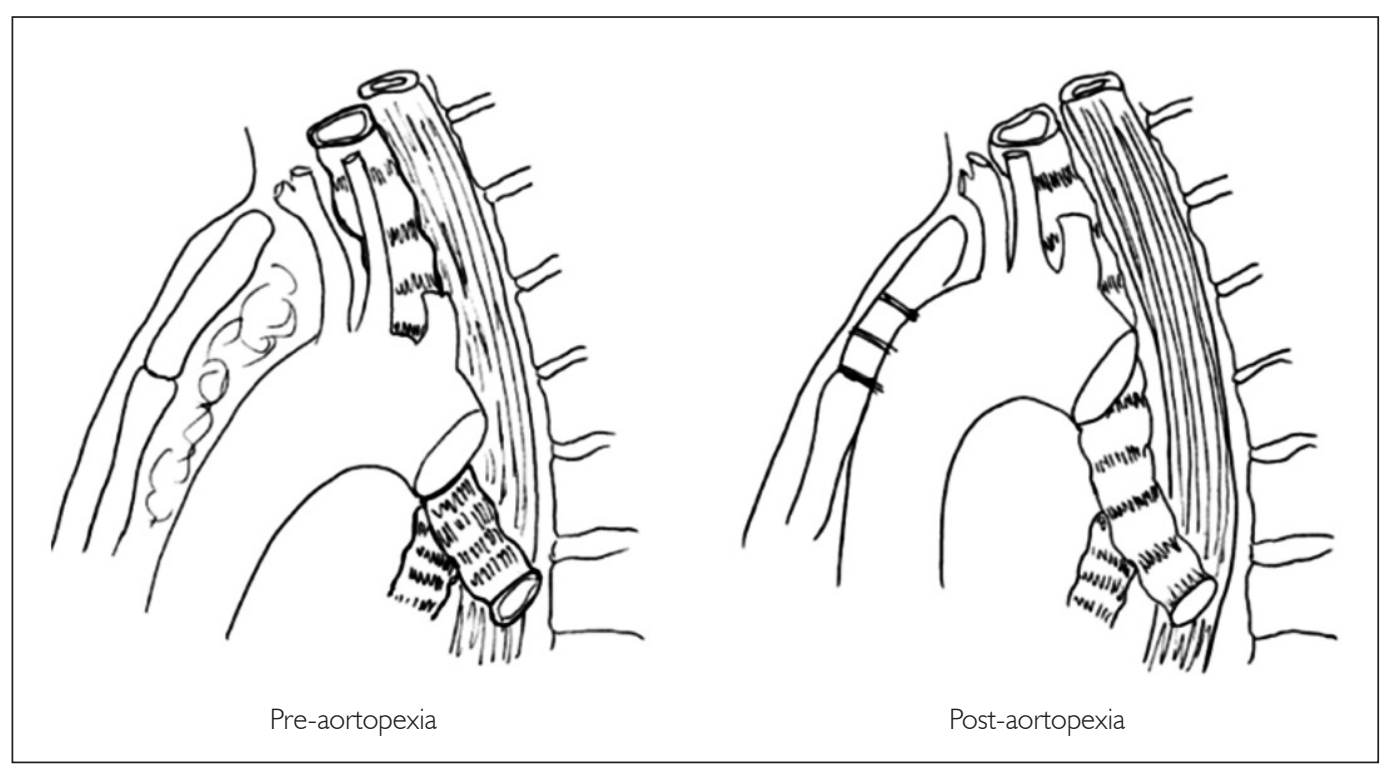

Figura 4. Aortopexia. 
Tabla 3. Evidencia en el manejo de la traqueobroncomalacia

\begin{tabular}{|llcc|}
\hline Terapia & Recomendación & Grado de recomendación & Calidad de la evidencia \\
$\beta 2$ agonistas & No de rutina & Moderada & Baja \\
\hline Corticoide inhalados y orales & No de rutina & Baja & Baja \\
\hline Antibióticos & En bronquitis prolongada & Baja & Baja \\
\hline Kinesioterapia & Recomendada & Baja & Baja \\
\hline Ciruǵa & En casos severos & Baja & Baja \\
\hline
\end{tabular}

grande en la medida que el niño crece. La factibilidad de remover el stent es importante ya que lo más probable es que la malacia se resuelva en la medida que el niño crece y se vuelva asintomático. Actualmente, el uso de stents está limitado a situaciones particulares en que otras terapias han fallado. Están en desarrollo stents reabsorbibles que podrían resolver los principales problemas de los dispositivos actuales y volver a situarlos como una excelente alternativa en el tratamiento de las malacias.

\section{Tratamiento según severidad}

- Leve (infecciones respiratorias recurrentes): Antibióticos, kinesioterapia respiratoria (nivel de evidencia $=>$ muy bajo).

- Moderada (obstrucción de vía aérea moderada persistente o severa intermitente): oxígenoterapia, CPAP o BiPAP durante el sueño (nivel de evidencia $=>$ muy bajo).

- Severa (obstrucción de vía aérea de riesgo vital, necesidad de cuidados intensivos e intubación): Cirugía (aortopexia, traqueostomía) (nivel de evidencia $=>$ muy bajo).

En la Tabla 3 se resume el nivel de e evidencia para otras opciones terapéuticas en TBM.

\section{PRONÓSTICO}

La mayoría de las lesiones tiende a mejorar con el tiempo y sólo un pequeño porcentaje permanece igual o empeora. No es posible predecir que lesiones no mejorarán con el tiempo con la evidencia disponible.

\section{REFERENCIAS}

I. Carden K, et al. Tracheomalacia and Tracheobronchomalacia in Children and Adults: An In-depth Review. Chest 2005; 127: 9841005.

2. Masters B. Congenital Airway Lesions and Lung Disease. Pediatr Clin N Am 2009; 56: 227-42.

3. Weinberger $M$, et al. Pseudo-Asthma: When Cough, Wheezing and Dyspnea Are Not Asthma. Pediatrics 2007; 120: 855-64.

4. McNamara V, et al. Tracheomalacia. Paediatr Respir Rev 2004; 5: 147-54.

5. Boogaard R, et al. Recombinant Human DNase in Children With Airway Malacia and Lower Respiratory Tract Infection. Pediatr Pulmonol 2009; 44: 962-9. 